\title{
Easily magnetic anomalies earthquake prediction
}

\author{
Min JIANG
}

Ningbo Polytechnic ; Zhejiang Ningbo 315800

\begin{abstract}
Low power consumption long time offset magnetic field detector (earthquake prediction) .The design of the hardware circuit of the magnetic field detector seismic geomagnetic acquisition and pre processing module mainly includes. Electronic compass, compass. monitoring device while the magnetic azimuth for monitoring and analyzing the object, GSM, but it can also be applied to other seismic precursor information analysis, such as earthquake precursory infrasound abnormality, only need infrasound abnormality intelligent sensor replace geomagnetic anomaly intelligent sensor, and modify the relevant parameters can be.
\end{abstract}

Key words: earthquake prediction; GSM circuit; LED; Electronic compass

\section{INTRODUCTION}

Earthquake is a common natural disaster, and the prediction of earthquake is a worldwide problem. Earthquake prediction is a worldwide famous puzzle and it is a necessity of scientists to do research on this significant thesis. In order to avoid the heavy losses caused by the earthquake, to find an effective method of imminent earthquake early warning is the hard direction of the earthquake researchers. In recent years, scientists have found that monitoring of the geomagnetic anomaly signal of imminent earthquake is one of effective methods of impending earthquake early warning. China suffers natural disasters, especially earthquake calamity, all the time from ancient times. If accurate prediction is possible, Chinese people may avoid that great loss of lives or fortune and they would not fear destructive earthquakes. We discussed the research background and status of magnetic measurements earthquake, proposed the design of earthquake early warning system on the basis of explore seismomagnetic relations. The working principle of the system is real time monitoring the earth's magnetic field vertical component $\mathrm{Z}$ and magnetic north point a. To give an accurate earthquake prediction is very difficult since that there is too much information relating to earthquake and scientists could hardly distinguish precursor from artificial interference or background noise. As a result, author choose magnetic methods as a breakthrough point after reading lots of inferences. It's possible to record geomagnetic data per second benefiting from the use of new instruments. In this paper, many methods are used to eliminate interference and then first difference method is used to analyze geomagnetic data. The frequency of anomalies gives more information than the amplitude of first difference of geomagnetic data and such operation could avoid unknown artificial inference that may existed and give a comparison with natural background.

Low power consumption long time offset magnetic field detector (earthquake prediction) consists of electronic compass, compass. Is based on such a fact: Beichuan middle school in 2008 May 12, afternoon 2:15 class time is before the Wenchuan earthquake 13 minutes. According to the recollections of grade two (1) class students, physics teacher of this class is "Electromagnetic" he brought the electromagnetic demonstrator and compass for teaching. At the beginning of the class, the electromagnetic demonstrator is not open, the teacher found on the table of the compass appears chaotic pendulum. Sitting in the middle of a row of students recalled that the teacher's compass is a bit like a bamboo dragonfly, a large pointer, with the vertical support vertical, the teacher found the compass is wrong, has been 
staring at the compass". "He took his fingers, the compass and turned again, the teacher again with a finger to stop the compass, to open the finger compass or chaos." Students recall, the teacher began to write on the blackboard, from time to time with the benefit of hindsight compass, students on the compass turn indiscriminately whispered together to discuss. The students, and so on for a long time did not stop to make everyone laugh. Is based on such a fact:

\section{THEORETICAL BASIS}

Overview of the earth's magnetic field: the earth's magnetic field is called the geomagnetic field. Geomagnetic field approximation to the one placed in the centre of the earth's magnetic dipole magnetic field, the magnetic dipole that geocentric magnetic dipole or geocentric dipole, magnetic lines of force distribution as shown in the fig.1. The earth's magnetic field is a stable magnetic field, the strength of about $0.5 \sim 0.6$ Gauss, because the magnetic field is a variety of different sources of magnetic field is the result of the superposition, so in terms of its nature, is divided into two parts: one is the main source of stable magnetic field within the solid earth, covers an area of magnetic field of more than $95 \%$; the second is main source to the variation of the magnetic field outside of the solid earth, the biggest change covers the magnetic field strength of $2 \% \sim 4 \%$.

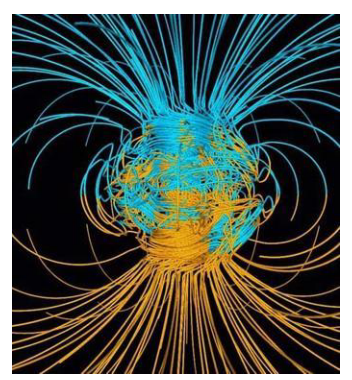

Fig.1 Schematic diagram of magnetic dipole magnetic field

In addition, the New York Times reported in 1858 November 11, before the earthquake in Setubal, Portugal, local only appeared in the compass abnormal phenomenon, even the mariner's compass pointer occur irregular circles of the fact. Before and after the two similar impending earthquake anomalies difference of 150 years, can explain the strong geomagnetic anomalies before the earthquake is not accidental.

The geomagnetic field is closely related to the structure of the earth's interior. A large number of observations and studies have found that the magnetic azimuth angle of the epicenter of the earthquake occurred in the area of the epicenter of the earthquake occurred a few minutes to a few days before the magnitude 5 or above. So in pre earthquake on the research of the magnetic azimuth anomaly information, not only to deepen knowledge and understanding of seismomagnetic effect, and probably become one of the important way of earthquake early warning.

In the determination of space electric field at the same time, choose to use the low power magnetic deviation detector consists of electronic compass, a compass. Using the special timing device opened 30 seconds per minute. Before the earthquake electromagnetic anomaly magnetoelectric effect is the main factor that caused by the earthquake, the earthquake electromagnetic anomaly before confirmed by a large number of examples. Use of the space electric field and the earth magnetic field changes in the design of earthquake prediction, the electric field, magnetic field parameters measurement is not necessary to enter the interior of the earth, technically easy to implement, low power consumption long time offset a major advantage of the detector is not the earth magnetic field debugging can work normally. The static when the power circuit is only $0.1 \sim 1 \mu \mathrm{A}$, through to 30 seconds per minute, while working current general $<10 \mathrm{~mA}$, so it is very save electricity, two new general 2 number No.5 cell. 


\section{HARDWARE}

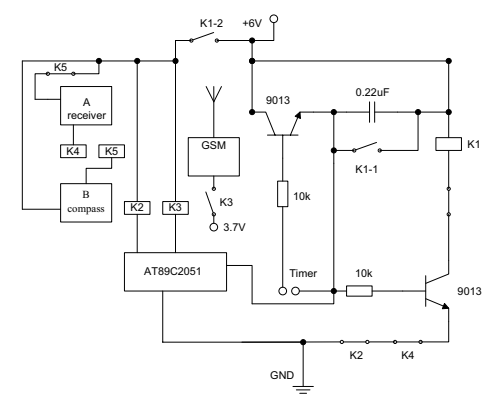

Fig.2 Earthquake prediction device hardware

When the remote alarm circuit in the normal electromagnetic GSM, remote automatic alarm when abnormal electromagnetic, alarm self-locking want to disarm by remote control after launch "output ff" command. A charge can alarm more than 200 times, is very convenient. GPRS remote monitoring device can also analysis level and the exact time of the earthquake epicentre of the earthquake. Most of the devastating earthquake in China. This device can know a few days to a few hours in advance before the earthquake electromagnetic anomaly can avoid many of the terrible disasters. Fig.2 Earthquake prediction device hardware.

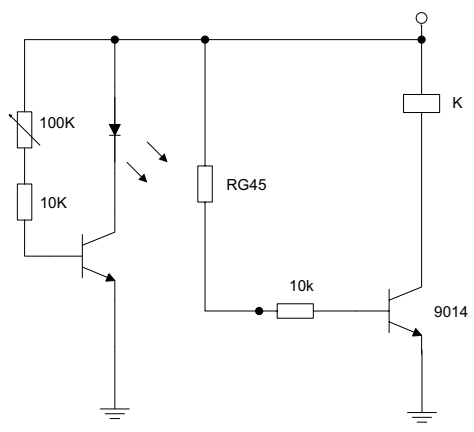

Fig.3 Earthquake prediction device LED Circuit

Used to increase the earth's magnetic field, is the switching element, open collector output contactless, long life. Fast switching speed and frequency band (DC to $100 \mathrm{kHz}$ ). Because the earth's magnetic field is very weak so about two ferrite rod enhanced, and achieved good results. DN6851 Hall components through T2, T1 amplification direct drive music integrated circuit, the circuit is connected 4 times per minute, time of about 10 seconds. Alarm when the magnetic field changes before the earthquake. The music integrated circuit is actually a large-scale CMOS (complementary symmetry metal oxide semiconductor integrated circuits English abbreviation) circuit, transistor best the power triode in $300 \mathrm{MW}$ silicon NPN type electrode dissipation power larger than that of the, 9013, 8050, 3DG12, 3DK4 and 3DX201 type requires current amplification factor of 100 . LED is the best choice of $5 \mathrm{~mm}$ red light emitting diodes. The $8 \Omega, 0.25 \mathrm{w}$ small caliber horn loudspeakers. The power supply is connected in series with two 5 numbers (to be equipped with a plastic cell rack), and the voltage is $3 \mathrm{~V}$.

\section{SOFTWARE}

The discrete wavelet transform (DWT) is a mathematical tool that transforms a data vector of length $\mathrm{M}$ into a different vector of the same length. It is based on recursive sums and differences of the vector components; the sum can be compared with the low frequency amplitude in the Fourier transform, and the differences with the high frequency amplitudes. It is similar to the discrete Fourier transform concerning the properties of orthogonality and invertibility, and the wavelets represent the basis vectors of the transform, like the sine and cosine functions represent the basis functions of the Fourier transform. One of the basic advantages of wavelets is that an event can be simultaneously described in the frequency domain as well as in the time domain, unlike the usual Fourier transform where an event is accurately described either in the frequency or in the time domain. This feature permits a multiresolution analysis of data with different behaviour on different scales. Fig.4 is Earthquake prediction MCU software. Signal sampling and wavelet transform are used to achieve single-chip software. In the work, the software is combined with the hardware circuit to realize the discrete wavelet transform. 


\begin{tabular}{|c|c|c|c|}
\hline & \multicolumn{2}{|l|}{ ORG } & \multirow[t]{2}{*}{$0000 \mathrm{H}$} \\
\hline & AJMP & START1 & \\
\hline & ORG & & $0030 \mathrm{H}$ \\
\hline SAME & $E Q U$ & & $4 \mathrm{EH}$ \\
\hline START1: & MOV & & $\mathrm{P} 1, \# 00 \mathrm{O}$ \\
\hline \multirow[t]{4}{*}{ START: } & MOV & & $\mathrm{A}, \mathrm{P} 3$ \\
\hline & ANL & & $\mathrm{A}, \# 1 \mathrm{FH}$ \\
\hline & CJNE & $\mathrm{A}, \# 1 \mathrm{FH}, \mathrm{S}$ & SHIY \\
\hline & AJMP & START1 & \\
\hline \multirow[t]{6}{*}{ SHIY: } & MOV & & SAME, A \\
\hline & LCALI & YS & \\
\hline & MOV & & $\mathrm{A}, \mathrm{P3}$ \\
\hline & ANL & & $\mathrm{A}, \# 1 \mathrm{FH}$ \\
\hline & CJNE & $\mathrm{A}, \# 1 \mathrm{FH}, \mathrm{S}$ & SHIY1 \\
\hline & AJMP & START1 & \\
\hline \multirow[t]{3}{*}{ SHIY1: } & CUNE & A, SAME, S & START1 \\
\hline & CUNE & $\mathrm{A}, \# 17 \mathrm{H}, \mathrm{N}$ & NEXT1 \\
\hline & AJMP & LEFT & \\
\hline \multirow[t]{2}{*}{ NEXT1: } & CUNE & $\mathrm{A}, \# 0 \mathrm{FH}, \mathrm{D}$ & NEXT2 \\
\hline & AJMP & RIGHT & \\
\hline \multirow[t]{2}{*}{ NEXT2: } & CUNE & $\mathrm{A}, \# 1 \mathrm{DH}, \mathrm{l}$ & NEXT3 \\
\hline & AJMP & EARGE & \\
\hline \multirow[t]{2}{*}{ NEXT3: } & CJNE & $\mathrm{A}, \# 1 \mathrm{EH}, \mathrm{D}$ & NEXT 4 \\
\hline & AJMP & BRAKE & \\
\hline
\end{tabular}

Fig.4 Earthquake prediction MCU software

The ordinary compass warning circuit and its working principle: watch movement is mainly used for circuit four times per minute is switched on, the red LED light per minute 30 seconds were detected, the MG45 (20k) photosensitive resistance for receiving the magnetic offset, if found abnormal driving a music integrated circuit work, two No.5 dry batteries are available for about one year time. The entire production cost is lower. Components and models see third parts. Welding should be paid special attention to, because the music integrated circuit CMOS circuit, so electric iron shell must to have a good grounding, also can dial to the power plug of the electric soldering iron using waste heat of the electric iron welding so that it can be avoid external field induced breakdown in a music integrated circuit, and cause permanent damage. Welding the electric iron power should not be more than $30 \mathrm{~W}$, and stay on the circuit board time should be as short as possible, each solder joint time generally do not exceed the $2 \mathrm{sec}$. Do not use flux soldering paste or paste, if necessary, after use must be welded oil clean.

Warning circuit of Electronic Compass: is mainly composed of DN6851 Hall element and now use it to abnormalities in the monitoring of the earth's magnetic field, the basic principle is using the switch type Hall sensor (Hall switch) is a new non contact integrated circuit switch, DN6851 has simple structure single, plastic shell, small volume. The combined use of two ferrite rod. The Hall effect is the production of a voltage difference (the Hall voltage) across an electrical conductor, transverse to an electric current in the conductor and a magnetic field perpendicular to the current. It was discovered by Edwin Hall in 1879. The Hall coefficient is defined as the ratio of the induced electric field to the product of the current density and the applied magnetic field. It is a characteristic of the material from which the conductor is made, since its value depends on the type, number, and properties of the charge carriers that constitute the current.

\section{SUMMARY}

Geomagnetic field is closely related to the interior structure of Earth. large number of observations and researches show that the angel of magnetic azimuth in epicentral area would be obvious anomaly within a few minutes to several days before an earthquake of magnitude 5 or over. Therefore, a research in abnormal information of the angel at an impending earthquake not only deepens the knowledge and understanding of seismomagnetic effect, but also probably become one of the important ways of earthquake early warning. Low power consumption long time offset magnetic field detector (earthquake prediction) .The design of the hardware circuit of the magnetic field detector seismic geomagnetic acquisition and pre processing module mainly includes. Electronic compass, compass. monitoring device while the magnetic azimuth for monitoring and analyzing the object, GSM, but it can also be applied to other seismic precursor information analysis, such as earthquake precursory infrasound abnormality, only need infrasound abnormality intelligent sensor replace geomagnetic anomaly intelligent sensor, and modify the relevant parameters can be.

\section{REFERENCES}

1. Min Jiang,2013.11 How to weave the 
electromagnetic spider web to predict earthquakes , 2013 3rd International Conference on Education and Education Management(EEM 2013), p546-551

2. Min Jiang,2014.1 Electromagnetic spider web Application in earthquake prediction, International Core Journal of Scientific Research \& Engineering Index,p111-114.

3. Min Jiang,2014.11 The Group of Electromagnetic spider web, Economic, Business Management and Education Innovation(EBMEI 2014),p287-291.

4. Min Jiang,2014.12 Low Power Consumption
Electromagnetic spider web, 2014 4th International Conference on Education and Education Management(EEM 2014), p74-78.

5. Min Jiang, Ping Yao Wang 2015.9 Simple space electric field and earth magnetic anomaly remote alarm system,2015 International Conference on Frontiers of Manufacturing Science and Measuring Technology, p452-458.

6. Min Jiang,2016.4 Application of 3 kinds of practical electromagnetic spiders in electromagnetic spider web. ICEICE 2016 02083. MATEC Web of Conferences 44. 